\title{
Modal Consequence Relations Extending S4.3: An Application of Projective Unification
}

\author{
Wojciech Dzik and Piotr Wojtylak
}

\begin{abstract}
We characterize all finitary consequence relations over $\mathbf{S 4 . 3}$, both syntactically, by exhibiting so-called (admissible) passive rules that extend the given logic, and semantically, by providing suitable strongly adequate classes of algebras. This is achieved by applying an earlier result stating that a modal $\operatorname{logic} L$ extending $\mathbf{S 4}$ has projective unification if and only if $L$ contains $\mathbf{S 4 . 3}$. In particular, we show that these consequence relations enjoy the strong finite model property, and are finitely based. In this way, we extend the known results by Bull and Fine, from logics, to consequence relations. We also show that the lattice of consequence relations over $\mathbf{S 4 . 3}$ (the lattice of quasivarieties of $\mathbf{S 4 . 3}$-algebras) is countable and distributive and it forms a Heyting algebra.
\end{abstract}

\section{Introduction}

Modal logics extending S4.3 form a subset of the lattice of all modal logics in which some theorems on particular logics were generalized to the whole area. Recall that $\mathbf{S 4 . 3}$ is an extension of $\mathbf{S 4}$ with the axiom $\square(\square \alpha \rightarrow \square \beta) \vee \square(\square \beta \rightarrow \square \alpha)$.

First, Bull [4] proved that every such logic has the finite model property, FMP. Next, Fine [11] showed that all modal logics extending $\mathbf{S 4 . 3}$ have the finite frame property, which is equivalent to the FMP, and that they all are finitely axiomatizable. Fine also characterized all such logics by means of finite quasilinear frames (chains of clusters), or lists. Hence he provided a semantic description of all modal logics extending $\mathbf{S 4 . 3}$.

Following this line of investigations, we lift these results from the level of theoremhood into the level of derivability. In other words, we extend these results from

Received November 30, 2012; accepted November 11, 2013

First published online July 19, 2016

2010 Mathematics Subject Classification: Primary 03B45, 08C15, 68T15; Secondary 06E25, 03B35

Keywords: admissible rules, consequence relations, projective unification, $\mathbf{S 4 . 3}$, structural completeness, quasivarieties

(C) 2016 by University of Notre Dame 10.1215/00294527-3636512 


\title{
Modal Consequence Relations Extending S4.3: An Application of Projective Unification
}

\author{
Wojciech Dzik and Piotr Wojtylak
}

\begin{abstract}
We characterize all finitary consequence relations over $\mathbf{S 4 . 3}$, both syntactically, by exhibiting so-called (admissible) passive rules that extend the given logic, and semantically, by providing suitable strongly adequate classes of algebras. This is achieved by applying an earlier result stating that a modal $\operatorname{logic} L$ extending $\mathbf{S 4}$ has projective unification if and only if $L$ contains $\mathbf{S 4 . 3}$. In particular, we show that these consequence relations enjoy the strong finite model property, and are finitely based. In this way, we extend the known results by Bull and Fine, from logics, to consequence relations. We also show that the lattice of consequence relations over $\mathbf{S 4 . 3}$ (the lattice of quasivarieties of $\mathbf{S 4 . 3}$-algebras) is countable and distributive and it forms a Heyting algebra.
\end{abstract}

\section{Introduction}

Modal logics extending S4.3 form a subset of the lattice of all modal logics in which some theorems on particular logics were generalized to the whole area. Recall that $\mathbf{S 4 . 3}$ is an extension of $\mathbf{S 4}$ with the axiom $\square(\square \alpha \rightarrow \square \beta) \vee \square(\square \beta \rightarrow \square \alpha)$.

First, Bull [4] proved that every such logic has the finite model property, FMP. Next, Fine [11] showed that all modal logics extending $\mathbf{S 4 . 3}$ have the finite frame property, which is equivalent to the FMP, and that they all are finitely axiomatizable. Fine also characterized all such logics by means of finite quasilinear frames (chains of clusters), or lists. Hence he provided a semantic description of all modal logics extending $\mathbf{S 4 . 3}$.

Following this line of investigations, we lift these results from the level of theoremhood into the level of derivability. In other words, we extend these results from

Received November 30, 2012; accepted November 11, 2013

First published online July 19, 2016

2010 Mathematics Subject Classification: Primary 03B45, 08C15, 68T15; Secondary 06E25, 03B35

Keywords: admissible rules, consequence relations, projective unification, $\mathbf{S 4 . 3}$, structural completeness, quasivarieties

(C) 2016 by University of Notre Dame 10.1215/00294527-3636512 


\title{
Modal Consequence Relations Extending S4.3: An Application of Projective Unification
}

\author{
Wojciech Dzik and Piotr Wojtylak
}

\begin{abstract}
We characterize all finitary consequence relations over $\mathbf{S 4 . 3}$, both syntactically, by exhibiting so-called (admissible) passive rules that extend the given logic, and semantically, by providing suitable strongly adequate classes of algebras. This is achieved by applying an earlier result stating that a modal $\operatorname{logic} L$ extending $\mathbf{S 4}$ has projective unification if and only if $L$ contains $\mathbf{S 4 . 3}$. In particular, we show that these consequence relations enjoy the strong finite model property, and are finitely based. In this way, we extend the known results by Bull and Fine, from logics, to consequence relations. We also show that the lattice of consequence relations over $\mathbf{S 4 . 3}$ (the lattice of quasivarieties of $\mathbf{S 4 . 3}$-algebras) is countable and distributive and it forms a Heyting algebra.
\end{abstract}

\section{Introduction}

Modal logics extending S4.3 form a subset of the lattice of all modal logics in which some theorems on particular logics were generalized to the whole area. Recall that $\mathbf{S 4 . 3}$ is an extension of $\mathbf{S 4}$ with the axiom $\square(\square \alpha \rightarrow \square \beta) \vee \square(\square \beta \rightarrow \square \alpha)$.

First, Bull [4] proved that every such logic has the finite model property, FMP. Next, Fine [11] showed that all modal logics extending $\mathbf{S 4 . 3}$ have the finite frame property, which is equivalent to the FMP, and that they all are finitely axiomatizable. Fine also characterized all such logics by means of finite quasilinear frames (chains of clusters), or lists. Hence he provided a semantic description of all modal logics extending $\mathbf{S 4 . 3}$.

Following this line of investigations, we lift these results from the level of theoremhood into the level of derivability. In other words, we extend these results from

Received November 30, 2012; accepted November 11, 2013

First published online July 19, 2016

2010 Mathematics Subject Classification: Primary 03B45, 08C15, 68T15; Secondary 06E25, 03B35

Keywords: admissible rules, consequence relations, projective unification, $\mathbf{S 4 . 3}$, structural completeness, quasivarieties

(C) 2016 by University of Notre Dame 10.1215/00294527-3636512 


\title{
Modal Consequence Relations Extending S4.3: An Application of Projective Unification
}

\author{
Wojciech Dzik and Piotr Wojtylak
}

\begin{abstract}
We characterize all finitary consequence relations over $\mathbf{S 4 . 3}$, both syntactically, by exhibiting so-called (admissible) passive rules that extend the given logic, and semantically, by providing suitable strongly adequate classes of algebras. This is achieved by applying an earlier result stating that a modal $\operatorname{logic} L$ extending $\mathbf{S 4}$ has projective unification if and only if $L$ contains $\mathbf{S 4 . 3}$. In particular, we show that these consequence relations enjoy the strong finite model property, and are finitely based. In this way, we extend the known results by Bull and Fine, from logics, to consequence relations. We also show that the lattice of consequence relations over $\mathbf{S 4 . 3}$ (the lattice of quasivarieties of $\mathbf{S 4 . 3}$-algebras) is countable and distributive and it forms a Heyting algebra.
\end{abstract}

\section{Introduction}

Modal logics extending S4.3 form a subset of the lattice of all modal logics in which some theorems on particular logics were generalized to the whole area. Recall that $\mathbf{S 4 . 3}$ is an extension of $\mathbf{S 4}$ with the axiom $\square(\square \alpha \rightarrow \square \beta) \vee \square(\square \beta \rightarrow \square \alpha)$.

First, Bull [4] proved that every such logic has the finite model property, FMP. Next, Fine [11] showed that all modal logics extending $\mathbf{S 4 . 3}$ have the finite frame property, which is equivalent to the FMP, and that they all are finitely axiomatizable. Fine also characterized all such logics by means of finite quasilinear frames (chains of clusters), or lists. Hence he provided a semantic description of all modal logics extending $\mathbf{S 4 . 3}$.

Following this line of investigations, we lift these results from the level of theoremhood into the level of derivability. In other words, we extend these results from

Received November 30, 2012; accepted November 11, 2013

First published online July 19, 2016

2010 Mathematics Subject Classification: Primary 03B45, 08C15, 68T15; Secondary 06E25, 03B35

Keywords: admissible rules, consequence relations, projective unification, $\mathbf{S 4 . 3}$, structural completeness, quasivarieties

(C) 2016 by University of Notre Dame 10.1215/00294527-3636512 


\title{
Modal Consequence Relations Extending S4.3: An Application of Projective Unification
}

\author{
Wojciech Dzik and Piotr Wojtylak
}

\begin{abstract}
We characterize all finitary consequence relations over $\mathbf{S 4 . 3}$, both syntactically, by exhibiting so-called (admissible) passive rules that extend the given logic, and semantically, by providing suitable strongly adequate classes of algebras. This is achieved by applying an earlier result stating that a modal $\operatorname{logic} L$ extending $\mathbf{S 4}$ has projective unification if and only if $L$ contains $\mathbf{S 4 . 3}$. In particular, we show that these consequence relations enjoy the strong finite model property, and are finitely based. In this way, we extend the known results by Bull and Fine, from logics, to consequence relations. We also show that the lattice of consequence relations over $\mathbf{S 4 . 3}$ (the lattice of quasivarieties of $\mathbf{S 4 . 3}$-algebras) is countable and distributive and it forms a Heyting algebra.
\end{abstract}

\section{Introduction}

Modal logics extending S4.3 form a subset of the lattice of all modal logics in which some theorems on particular logics were generalized to the whole area. Recall that $\mathbf{S 4 . 3}$ is an extension of $\mathbf{S 4}$ with the axiom $\square(\square \alpha \rightarrow \square \beta) \vee \square(\square \beta \rightarrow \square \alpha)$.

First, Bull [4] proved that every such logic has the finite model property, FMP. Next, Fine [11] showed that all modal logics extending $\mathbf{S 4 . 3}$ have the finite frame property, which is equivalent to the FMP, and that they all are finitely axiomatizable. Fine also characterized all such logics by means of finite quasilinear frames (chains of clusters), or lists. Hence he provided a semantic description of all modal logics extending $\mathbf{S 4 . 3}$.

Following this line of investigations, we lift these results from the level of theoremhood into the level of derivability. In other words, we extend these results from

Received November 30, 2012; accepted November 11, 2013

First published online July 19, 2016

2010 Mathematics Subject Classification: Primary 03B45, 08C15, 68T15; Secondary 06E25, 03B35

Keywords: admissible rules, consequence relations, projective unification, $\mathbf{S 4 . 3}$, structural completeness, quasivarieties

(C) 2016 by University of Notre Dame 10.1215/00294527-3636512 


\title{
Modal Consequence Relations Extending S4.3: An Application of Projective Unification
}

\author{
Wojciech Dzik and Piotr Wojtylak
}

\begin{abstract}
We characterize all finitary consequence relations over $\mathbf{S 4 . 3}$, both syntactically, by exhibiting so-called (admissible) passive rules that extend the given logic, and semantically, by providing suitable strongly adequate classes of algebras. This is achieved by applying an earlier result stating that a modal $\operatorname{logic} L$ extending $\mathbf{S 4}$ has projective unification if and only if $L$ contains $\mathbf{S 4 . 3}$. In particular, we show that these consequence relations enjoy the strong finite model property, and are finitely based. In this way, we extend the known results by Bull and Fine, from logics, to consequence relations. We also show that the lattice of consequence relations over $\mathbf{S 4 . 3}$ (the lattice of quasivarieties of $\mathbf{S 4 . 3}$-algebras) is countable and distributive and it forms a Heyting algebra.
\end{abstract}

\section{Introduction}

Modal logics extending S4.3 form a subset of the lattice of all modal logics in which some theorems on particular logics were generalized to the whole area. Recall that $\mathbf{S 4 . 3}$ is an extension of $\mathbf{S 4}$ with the axiom $\square(\square \alpha \rightarrow \square \beta) \vee \square(\square \beta \rightarrow \square \alpha)$.

First, Bull [4] proved that every such logic has the finite model property, FMP. Next, Fine [11] showed that all modal logics extending $\mathbf{S 4 . 3}$ have the finite frame property, which is equivalent to the FMP, and that they all are finitely axiomatizable. Fine also characterized all such logics by means of finite quasilinear frames (chains of clusters), or lists. Hence he provided a semantic description of all modal logics extending $\mathbf{S 4 . 3}$.

Following this line of investigations, we lift these results from the level of theoremhood into the level of derivability. In other words, we extend these results from

Received November 30, 2012; accepted November 11, 2013

First published online July 19, 2016

2010 Mathematics Subject Classification: Primary 03B45, 08C15, 68T15; Secondary 06E25, 03B35

Keywords: admissible rules, consequence relations, projective unification, $\mathbf{S 4 . 3}$, structural completeness, quasivarieties

(C) 2016 by University of Notre Dame 10.1215/00294527-3636512 


\title{
Modal Consequence Relations Extending S4.3: An Application of Projective Unification
}

\author{
Wojciech Dzik and Piotr Wojtylak
}

\begin{abstract}
We characterize all finitary consequence relations over $\mathbf{S 4 . 3}$, both syntactically, by exhibiting so-called (admissible) passive rules that extend the given logic, and semantically, by providing suitable strongly adequate classes of algebras. This is achieved by applying an earlier result stating that a modal $\operatorname{logic} L$ extending $\mathbf{S 4}$ has projective unification if and only if $L$ contains $\mathbf{S 4 . 3}$. In particular, we show that these consequence relations enjoy the strong finite model property, and are finitely based. In this way, we extend the known results by Bull and Fine, from logics, to consequence relations. We also show that the lattice of consequence relations over $\mathbf{S 4 . 3}$ (the lattice of quasivarieties of $\mathbf{S 4 . 3}$-algebras) is countable and distributive and it forms a Heyting algebra.
\end{abstract}

\section{Introduction}

Modal logics extending S4.3 form a subset of the lattice of all modal logics in which some theorems on particular logics were generalized to the whole area. Recall that $\mathbf{S 4 . 3}$ is an extension of $\mathbf{S 4}$ with the axiom $\square(\square \alpha \rightarrow \square \beta) \vee \square(\square \beta \rightarrow \square \alpha)$.

First, Bull [4] proved that every such logic has the finite model property, FMP. Next, Fine [11] showed that all modal logics extending $\mathbf{S 4 . 3}$ have the finite frame property, which is equivalent to the FMP, and that they all are finitely axiomatizable. Fine also characterized all such logics by means of finite quasilinear frames (chains of clusters), or lists. Hence he provided a semantic description of all modal logics extending $\mathbf{S 4 . 3}$.

Following this line of investigations, we lift these results from the level of theoremhood into the level of derivability. In other words, we extend these results from

Received November 30, 2012; accepted November 11, 2013

First published online July 19, 2016

2010 Mathematics Subject Classification: Primary 03B45, 08C15, 68T15; Secondary 06E25, 03B35

Keywords: admissible rules, consequence relations, projective unification, $\mathbf{S 4 . 3}$, structural completeness, quasivarieties

(C) 2016 by University of Notre Dame 10.1215/00294527-3636512 


\title{
Modal Consequence Relations Extending S4.3: An Application of Projective Unification
}

\author{
Wojciech Dzik and Piotr Wojtylak
}

\begin{abstract}
We characterize all finitary consequence relations over $\mathbf{S 4 . 3}$, both syntactically, by exhibiting so-called (admissible) passive rules that extend the given logic, and semantically, by providing suitable strongly adequate classes of algebras. This is achieved by applying an earlier result stating that a modal $\operatorname{logic} L$ extending $\mathbf{S 4}$ has projective unification if and only if $L$ contains $\mathbf{S 4 . 3}$. In particular, we show that these consequence relations enjoy the strong finite model property, and are finitely based. In this way, we extend the known results by Bull and Fine, from logics, to consequence relations. We also show that the lattice of consequence relations over $\mathbf{S 4 . 3}$ (the lattice of quasivarieties of $\mathbf{S 4 . 3}$-algebras) is countable and distributive and it forms a Heyting algebra.
\end{abstract}

\section{Introduction}

Modal logics extending S4.3 form a subset of the lattice of all modal logics in which some theorems on particular logics were generalized to the whole area. Recall that $\mathbf{S 4 . 3}$ is an extension of $\mathbf{S 4}$ with the axiom $\square(\square \alpha \rightarrow \square \beta) \vee \square(\square \beta \rightarrow \square \alpha)$.

First, Bull [4] proved that every such logic has the finite model property, FMP. Next, Fine [11] showed that all modal logics extending $\mathbf{S 4 . 3}$ have the finite frame property, which is equivalent to the FMP, and that they all are finitely axiomatizable. Fine also characterized all such logics by means of finite quasilinear frames (chains of clusters), or lists. Hence he provided a semantic description of all modal logics extending $\mathbf{S 4 . 3}$.

Following this line of investigations, we lift these results from the level of theoremhood into the level of derivability. In other words, we extend these results from

Received November 30, 2012; accepted November 11, 2013

First published online July 19, 2016

2010 Mathematics Subject Classification: Primary 03B45, 08C15, 68T15; Secondary 06E25, 03B35

Keywords: admissible rules, consequence relations, projective unification, $\mathbf{S 4 . 3}$, structural completeness, quasivarieties

(C) 2016 by University of Notre Dame 10.1215/00294527-3636512 


\title{
Modal Consequence Relations Extending S4.3: An Application of Projective Unification
}

\author{
Wojciech Dzik and Piotr Wojtylak
}

\begin{abstract}
We characterize all finitary consequence relations over $\mathbf{S 4 . 3}$, both syntactically, by exhibiting so-called (admissible) passive rules that extend the given logic, and semantically, by providing suitable strongly adequate classes of algebras. This is achieved by applying an earlier result stating that a modal $\operatorname{logic} L$ extending $\mathbf{S 4}$ has projective unification if and only if $L$ contains $\mathbf{S 4 . 3}$. In particular, we show that these consequence relations enjoy the strong finite model property, and are finitely based. In this way, we extend the known results by Bull and Fine, from logics, to consequence relations. We also show that the lattice of consequence relations over $\mathbf{S 4 . 3}$ (the lattice of quasivarieties of $\mathbf{S 4 . 3}$-algebras) is countable and distributive and it forms a Heyting algebra.
\end{abstract}

\section{Introduction}

Modal logics extending S4.3 form a subset of the lattice of all modal logics in which some theorems on particular logics were generalized to the whole area. Recall that $\mathbf{S 4 . 3}$ is an extension of $\mathbf{S 4}$ with the axiom $\square(\square \alpha \rightarrow \square \beta) \vee \square(\square \beta \rightarrow \square \alpha)$.

First, Bull [4] proved that every such logic has the finite model property, FMP. Next, Fine [11] showed that all modal logics extending $\mathbf{S 4 . 3}$ have the finite frame property, which is equivalent to the FMP, and that they all are finitely axiomatizable. Fine also characterized all such logics by means of finite quasilinear frames (chains of clusters), or lists. Hence he provided a semantic description of all modal logics extending $\mathbf{S 4 . 3}$.

Following this line of investigations, we lift these results from the level of theoremhood into the level of derivability. In other words, we extend these results from

Received November 30, 2012; accepted November 11, 2013

First published online July 19, 2016

2010 Mathematics Subject Classification: Primary 03B45, 08C15, 68T15; Secondary 06E25, 03B35

Keywords: admissible rules, consequence relations, projective unification, $\mathbf{S 4 . 3}$, structural completeness, quasivarieties

(C) 2016 by University of Notre Dame 10.1215/00294527-3636512 


\title{
Modal Consequence Relations Extending S4.3: An Application of Projective Unification
}

\author{
Wojciech Dzik and Piotr Wojtylak
}

\begin{abstract}
We characterize all finitary consequence relations over $\mathbf{S 4 . 3}$, both syntactically, by exhibiting so-called (admissible) passive rules that extend the given logic, and semantically, by providing suitable strongly adequate classes of algebras. This is achieved by applying an earlier result stating that a modal $\operatorname{logic} L$ extending $\mathbf{S 4}$ has projective unification if and only if $L$ contains $\mathbf{S 4 . 3}$. In particular, we show that these consequence relations enjoy the strong finite model property, and are finitely based. In this way, we extend the known results by Bull and Fine, from logics, to consequence relations. We also show that the lattice of consequence relations over $\mathbf{S 4 . 3}$ (the lattice of quasivarieties of $\mathbf{S 4 . 3}$-algebras) is countable and distributive and it forms a Heyting algebra.
\end{abstract}

\section{Introduction}

Modal logics extending S4.3 form a subset of the lattice of all modal logics in which some theorems on particular logics were generalized to the whole area. Recall that $\mathbf{S 4 . 3}$ is an extension of $\mathbf{S 4}$ with the axiom $\square(\square \alpha \rightarrow \square \beta) \vee \square(\square \beta \rightarrow \square \alpha)$.

First, Bull [4] proved that every such logic has the finite model property, FMP. Next, Fine [11] showed that all modal logics extending $\mathbf{S 4 . 3}$ have the finite frame property, which is equivalent to the FMP, and that they all are finitely axiomatizable. Fine also characterized all such logics by means of finite quasilinear frames (chains of clusters), or lists. Hence he provided a semantic description of all modal logics extending $\mathbf{S 4 . 3}$.

Following this line of investigations, we lift these results from the level of theoremhood into the level of derivability. In other words, we extend these results from

Received November 30, 2012; accepted November 11, 2013

First published online July 19, 2016

2010 Mathematics Subject Classification: Primary 03B45, 08C15, 68T15; Secondary 06E25, 03B35

Keywords: admissible rules, consequence relations, projective unification, $\mathbf{S 4 . 3}$, structural completeness, quasivarieties

(C) 2016 by University of Notre Dame 10.1215/00294527-3636512 


\title{
Modal Consequence Relations Extending S4.3: An Application of Projective Unification
}

\author{
Wojciech Dzik and Piotr Wojtylak
}

\begin{abstract}
We characterize all finitary consequence relations over $\mathbf{S 4 . 3}$, both syntactically, by exhibiting so-called (admissible) passive rules that extend the given logic, and semantically, by providing suitable strongly adequate classes of algebras. This is achieved by applying an earlier result stating that a modal $\operatorname{logic} L$ extending $\mathbf{S 4}$ has projective unification if and only if $L$ contains $\mathbf{S 4 . 3}$. In particular, we show that these consequence relations enjoy the strong finite model property, and are finitely based. In this way, we extend the known results by Bull and Fine, from logics, to consequence relations. We also show that the lattice of consequence relations over $\mathbf{S 4 . 3}$ (the lattice of quasivarieties of $\mathbf{S 4 . 3}$-algebras) is countable and distributive and it forms a Heyting algebra.
\end{abstract}

\section{Introduction}

Modal logics extending S4.3 form a subset of the lattice of all modal logics in which some theorems on particular logics were generalized to the whole area. Recall that $\mathbf{S 4 . 3}$ is an extension of $\mathbf{S 4}$ with the axiom $\square(\square \alpha \rightarrow \square \beta) \vee \square(\square \beta \rightarrow \square \alpha)$.

First, Bull [4] proved that every such logic has the finite model property, FMP. Next, Fine [11] showed that all modal logics extending $\mathbf{S 4 . 3}$ have the finite frame property, which is equivalent to the FMP, and that they all are finitely axiomatizable. Fine also characterized all such logics by means of finite quasilinear frames (chains of clusters), or lists. Hence he provided a semantic description of all modal logics extending $\mathbf{S 4 . 3}$.

Following this line of investigations, we lift these results from the level of theoremhood into the level of derivability. In other words, we extend these results from

Received November 30, 2012; accepted November 11, 2013

First published online July 19, 2016

2010 Mathematics Subject Classification: Primary 03B45, 08C15, 68T15; Secondary 06E25, 03B35

Keywords: admissible rules, consequence relations, projective unification, $\mathbf{S 4 . 3}$, structural completeness, quasivarieties

(C) 2016 by University of Notre Dame 10.1215/00294527-3636512 


\title{
Modal Consequence Relations Extending S4.3: An Application of Projective Unification
}

\author{
Wojciech Dzik and Piotr Wojtylak
}

\begin{abstract}
We characterize all finitary consequence relations over $\mathbf{S 4 . 3}$, both syntactically, by exhibiting so-called (admissible) passive rules that extend the given logic, and semantically, by providing suitable strongly adequate classes of algebras. This is achieved by applying an earlier result stating that a modal $\operatorname{logic} L$ extending $\mathbf{S 4}$ has projective unification if and only if $L$ contains $\mathbf{S 4 . 3}$. In particular, we show that these consequence relations enjoy the strong finite model property, and are finitely based. In this way, we extend the known results by Bull and Fine, from logics, to consequence relations. We also show that the lattice of consequence relations over $\mathbf{S 4 . 3}$ (the lattice of quasivarieties of $\mathbf{S 4 . 3}$-algebras) is countable and distributive and it forms a Heyting algebra.
\end{abstract}

\section{Introduction}

Modal logics extending S4.3 form a subset of the lattice of all modal logics in which some theorems on particular logics were generalized to the whole area. Recall that $\mathbf{S 4 . 3}$ is an extension of $\mathbf{S 4}$ with the axiom $\square(\square \alpha \rightarrow \square \beta) \vee \square(\square \beta \rightarrow \square \alpha)$.

First, Bull [4] proved that every such logic has the finite model property, FMP. Next, Fine [11] showed that all modal logics extending $\mathbf{S 4 . 3}$ have the finite frame property, which is equivalent to the FMP, and that they all are finitely axiomatizable. Fine also characterized all such logics by means of finite quasilinear frames (chains of clusters), or lists. Hence he provided a semantic description of all modal logics extending $\mathbf{S 4 . 3}$.

Following this line of investigations, we lift these results from the level of theoremhood into the level of derivability. In other words, we extend these results from

Received November 30, 2012; accepted November 11, 2013

First published online July 19, 2016

2010 Mathematics Subject Classification: Primary 03B45, 08C15, 68T15; Secondary 06E25, 03B35

Keywords: admissible rules, consequence relations, projective unification, $\mathbf{S 4 . 3}$, structural completeness, quasivarieties

(C) 2016 by University of Notre Dame 10.1215/00294527-3636512 


\title{
Modal Consequence Relations Extending S4.3: An Application of Projective Unification
}

\author{
Wojciech Dzik and Piotr Wojtylak
}

\begin{abstract}
We characterize all finitary consequence relations over $\mathbf{S 4 . 3}$, both syntactically, by exhibiting so-called (admissible) passive rules that extend the given logic, and semantically, by providing suitable strongly adequate classes of algebras. This is achieved by applying an earlier result stating that a modal $\operatorname{logic} L$ extending $\mathbf{S 4}$ has projective unification if and only if $L$ contains $\mathbf{S 4 . 3}$. In particular, we show that these consequence relations enjoy the strong finite model property, and are finitely based. In this way, we extend the known results by Bull and Fine, from logics, to consequence relations. We also show that the lattice of consequence relations over $\mathbf{S 4 . 3}$ (the lattice of quasivarieties of $\mathbf{S 4 . 3}$-algebras) is countable and distributive and it forms a Heyting algebra.
\end{abstract}

\section{Introduction}

Modal logics extending S4.3 form a subset of the lattice of all modal logics in which some theorems on particular logics were generalized to the whole area. Recall that $\mathbf{S 4 . 3}$ is an extension of $\mathbf{S 4}$ with the axiom $\square(\square \alpha \rightarrow \square \beta) \vee \square(\square \beta \rightarrow \square \alpha)$.

First, Bull [4] proved that every such logic has the finite model property, FMP. Next, Fine [11] showed that all modal logics extending $\mathbf{S 4 . 3}$ have the finite frame property, which is equivalent to the FMP, and that they all are finitely axiomatizable. Fine also characterized all such logics by means of finite quasilinear frames (chains of clusters), or lists. Hence he provided a semantic description of all modal logics extending $\mathbf{S 4 . 3}$.

Following this line of investigations, we lift these results from the level of theoremhood into the level of derivability. In other words, we extend these results from

Received November 30, 2012; accepted November 11, 2013

First published online July 19, 2016

2010 Mathematics Subject Classification: Primary 03B45, 08C15, 68T15; Secondary 06E25, 03B35

Keywords: admissible rules, consequence relations, projective unification, $\mathbf{S 4 . 3}$, structural completeness, quasivarieties

(C) 2016 by University of Notre Dame 10.1215/00294527-3636512 


\title{
Modal Consequence Relations Extending S4.3: An Application of Projective Unification
}

\author{
Wojciech Dzik and Piotr Wojtylak
}

\begin{abstract}
We characterize all finitary consequence relations over $\mathbf{S 4 . 3}$, both syntactically, by exhibiting so-called (admissible) passive rules that extend the given logic, and semantically, by providing suitable strongly adequate classes of algebras. This is achieved by applying an earlier result stating that a modal $\operatorname{logic} L$ extending $\mathbf{S 4}$ has projective unification if and only if $L$ contains $\mathbf{S 4 . 3}$. In particular, we show that these consequence relations enjoy the strong finite model property, and are finitely based. In this way, we extend the known results by Bull and Fine, from logics, to consequence relations. We also show that the lattice of consequence relations over $\mathbf{S 4 . 3}$ (the lattice of quasivarieties of $\mathbf{S 4 . 3}$-algebras) is countable and distributive and it forms a Heyting algebra.
\end{abstract}

\section{Introduction}

Modal logics extending S4.3 form a subset of the lattice of all modal logics in which some theorems on particular logics were generalized to the whole area. Recall that $\mathbf{S 4 . 3}$ is an extension of $\mathbf{S 4}$ with the axiom $\square(\square \alpha \rightarrow \square \beta) \vee \square(\square \beta \rightarrow \square \alpha)$.

First, Bull [4] proved that every such logic has the finite model property, FMP. Next, Fine [11] showed that all modal logics extending $\mathbf{S 4 . 3}$ have the finite frame property, which is equivalent to the FMP, and that they all are finitely axiomatizable. Fine also characterized all such logics by means of finite quasilinear frames (chains of clusters), or lists. Hence he provided a semantic description of all modal logics extending $\mathbf{S 4 . 3}$.

Following this line of investigations, we lift these results from the level of theoremhood into the level of derivability. In other words, we extend these results from

Received November 30, 2012; accepted November 11, 2013

First published online July 19, 2016

2010 Mathematics Subject Classification: Primary 03B45, 08C15, 68T15; Secondary 06E25, 03B35

Keywords: admissible rules, consequence relations, projective unification, $\mathbf{S 4 . 3}$, structural completeness, quasivarieties

(C) 2016 by University of Notre Dame 10.1215/00294527-3636512 


\title{
Modal Consequence Relations Extending S4.3: An Application of Projective Unification
}

\author{
Wojciech Dzik and Piotr Wojtylak
}

\begin{abstract}
We characterize all finitary consequence relations over $\mathbf{S 4 . 3}$, both syntactically, by exhibiting so-called (admissible) passive rules that extend the given logic, and semantically, by providing suitable strongly adequate classes of algebras. This is achieved by applying an earlier result stating that a modal $\operatorname{logic} L$ extending $\mathbf{S 4}$ has projective unification if and only if $L$ contains $\mathbf{S 4 . 3}$. In particular, we show that these consequence relations enjoy the strong finite model property, and are finitely based. In this way, we extend the known results by Bull and Fine, from logics, to consequence relations. We also show that the lattice of consequence relations over $\mathbf{S 4 . 3}$ (the lattice of quasivarieties of $\mathbf{S 4 . 3}$-algebras) is countable and distributive and it forms a Heyting algebra.
\end{abstract}

\section{Introduction}

Modal logics extending S4.3 form a subset of the lattice of all modal logics in which some theorems on particular logics were generalized to the whole area. Recall that $\mathbf{S 4 . 3}$ is an extension of $\mathbf{S 4}$ with the axiom $\square(\square \alpha \rightarrow \square \beta) \vee \square(\square \beta \rightarrow \square \alpha)$.

First, Bull [4] proved that every such logic has the finite model property, FMP. Next, Fine [11] showed that all modal logics extending $\mathbf{S 4 . 3}$ have the finite frame property, which is equivalent to the FMP, and that they all are finitely axiomatizable. Fine also characterized all such logics by means of finite quasilinear frames (chains of clusters), or lists. Hence he provided a semantic description of all modal logics extending $\mathbf{S 4 . 3}$.

Following this line of investigations, we lift these results from the level of theoremhood into the level of derivability. In other words, we extend these results from

Received November 30, 2012; accepted November 11, 2013

First published online July 19, 2016

2010 Mathematics Subject Classification: Primary 03B45, 08C15, 68T15; Secondary 06E25, 03B35

Keywords: admissible rules, consequence relations, projective unification, $\mathbf{S 4 . 3}$, structural completeness, quasivarieties

(C) 2016 by University of Notre Dame 10.1215/00294527-3636512 


\title{
Modal Consequence Relations Extending S4.3: An Application of Projective Unification
}

\author{
Wojciech Dzik and Piotr Wojtylak
}

\begin{abstract}
We characterize all finitary consequence relations over $\mathbf{S 4 . 3}$, both syntactically, by exhibiting so-called (admissible) passive rules that extend the given logic, and semantically, by providing suitable strongly adequate classes of algebras. This is achieved by applying an earlier result stating that a modal $\operatorname{logic} L$ extending $\mathbf{S 4}$ has projective unification if and only if $L$ contains $\mathbf{S 4 . 3}$. In particular, we show that these consequence relations enjoy the strong finite model property, and are finitely based. In this way, we extend the known results by Bull and Fine, from logics, to consequence relations. We also show that the lattice of consequence relations over $\mathbf{S 4 . 3}$ (the lattice of quasivarieties of $\mathbf{S 4 . 3}$-algebras) is countable and distributive and it forms a Heyting algebra.
\end{abstract}

\section{Introduction}

Modal logics extending S4.3 form a subset of the lattice of all modal logics in which some theorems on particular logics were generalized to the whole area. Recall that $\mathbf{S 4 . 3}$ is an extension of $\mathbf{S 4}$ with the axiom $\square(\square \alpha \rightarrow \square \beta) \vee \square(\square \beta \rightarrow \square \alpha)$.

First, Bull [4] proved that every such logic has the finite model property, FMP. Next, Fine [11] showed that all modal logics extending $\mathbf{S 4 . 3}$ have the finite frame property, which is equivalent to the FMP, and that they all are finitely axiomatizable. Fine also characterized all such logics by means of finite quasilinear frames (chains of clusters), or lists. Hence he provided a semantic description of all modal logics extending $\mathbf{S 4 . 3}$.

Following this line of investigations, we lift these results from the level of theoremhood into the level of derivability. In other words, we extend these results from

Received November 30, 2012; accepted November 11, 2013

First published online July 19, 2016

2010 Mathematics Subject Classification: Primary 03B45, 08C15, 68T15; Secondary 06E25, 03B35

Keywords: admissible rules, consequence relations, projective unification, $\mathbf{S 4 . 3}$, structural completeness, quasivarieties

(C) 2016 by University of Notre Dame 10.1215/00294527-3636512 


\title{
Modal Consequence Relations Extending S4.3: An Application of Projective Unification
}

\author{
Wojciech Dzik and Piotr Wojtylak
}

\begin{abstract}
We characterize all finitary consequence relations over $\mathbf{S 4 . 3}$, both syntactically, by exhibiting so-called (admissible) passive rules that extend the given logic, and semantically, by providing suitable strongly adequate classes of algebras. This is achieved by applying an earlier result stating that a modal $\operatorname{logic} L$ extending $\mathbf{S 4}$ has projective unification if and only if $L$ contains $\mathbf{S 4 . 3}$. In particular, we show that these consequence relations enjoy the strong finite model property, and are finitely based. In this way, we extend the known results by Bull and Fine, from logics, to consequence relations. We also show that the lattice of consequence relations over $\mathbf{S 4 . 3}$ (the lattice of quasivarieties of $\mathbf{S 4 . 3}$-algebras) is countable and distributive and it forms a Heyting algebra.
\end{abstract}

\section{Introduction}

Modal logics extending S4.3 form a subset of the lattice of all modal logics in which some theorems on particular logics were generalized to the whole area. Recall that $\mathbf{S 4 . 3}$ is an extension of $\mathbf{S 4}$ with the axiom $\square(\square \alpha \rightarrow \square \beta) \vee \square(\square \beta \rightarrow \square \alpha)$.

First, Bull [4] proved that every such logic has the finite model property, FMP. Next, Fine [11] showed that all modal logics extending $\mathbf{S 4 . 3}$ have the finite frame property, which is equivalent to the FMP, and that they all are finitely axiomatizable. Fine also characterized all such logics by means of finite quasilinear frames (chains of clusters), or lists. Hence he provided a semantic description of all modal logics extending $\mathbf{S 4 . 3}$.

Following this line of investigations, we lift these results from the level of theoremhood into the level of derivability. In other words, we extend these results from

Received November 30, 2012; accepted November 11, 2013

First published online July 19, 2016

2010 Mathematics Subject Classification: Primary 03B45, 08C15, 68T15; Secondary 06E25, 03B35

Keywords: admissible rules, consequence relations, projective unification, $\mathbf{S 4 . 3}$, structural completeness, quasivarieties

(C) 2016 by University of Notre Dame 10.1215/00294527-3636512 


\title{
Modal Consequence Relations Extending S4.3: An Application of Projective Unification
}

\author{
Wojciech Dzik and Piotr Wojtylak
}

\begin{abstract}
We characterize all finitary consequence relations over $\mathbf{S 4 . 3}$, both syntactically, by exhibiting so-called (admissible) passive rules that extend the given logic, and semantically, by providing suitable strongly adequate classes of algebras. This is achieved by applying an earlier result stating that a modal $\operatorname{logic} L$ extending $\mathbf{S 4}$ has projective unification if and only if $L$ contains $\mathbf{S 4 . 3}$. In particular, we show that these consequence relations enjoy the strong finite model property, and are finitely based. In this way, we extend the known results by Bull and Fine, from logics, to consequence relations. We also show that the lattice of consequence relations over $\mathbf{S 4 . 3}$ (the lattice of quasivarieties of $\mathbf{S 4 . 3}$-algebras) is countable and distributive and it forms a Heyting algebra.
\end{abstract}

\section{Introduction}

Modal logics extending S4.3 form a subset of the lattice of all modal logics in which some theorems on particular logics were generalized to the whole area. Recall that $\mathbf{S 4 . 3}$ is an extension of $\mathbf{S 4}$ with the axiom $\square(\square \alpha \rightarrow \square \beta) \vee \square(\square \beta \rightarrow \square \alpha)$.

First, Bull [4] proved that every such logic has the finite model property, FMP. Next, Fine [11] showed that all modal logics extending $\mathbf{S 4 . 3}$ have the finite frame property, which is equivalent to the FMP, and that they all are finitely axiomatizable. Fine also characterized all such logics by means of finite quasilinear frames (chains of clusters), or lists. Hence he provided a semantic description of all modal logics extending $\mathbf{S 4 . 3}$.

Following this line of investigations, we lift these results from the level of theoremhood into the level of derivability. In other words, we extend these results from

Received November 30, 2012; accepted November 11, 2013

First published online July 19, 2016

2010 Mathematics Subject Classification: Primary 03B45, 08C15, 68T15; Secondary 06E25, 03B35

Keywords: admissible rules, consequence relations, projective unification, $\mathbf{S 4 . 3}$, structural completeness, quasivarieties

(C) 2016 by University of Notre Dame 10.1215/00294527-3636512 


\title{
Modal Consequence Relations Extending S4.3: An Application of Projective Unification
}

\author{
Wojciech Dzik and Piotr Wojtylak
}

\begin{abstract}
We characterize all finitary consequence relations over $\mathbf{S 4 . 3}$, both syntactically, by exhibiting so-called (admissible) passive rules that extend the given logic, and semantically, by providing suitable strongly adequate classes of algebras. This is achieved by applying an earlier result stating that a modal $\operatorname{logic} L$ extending $\mathbf{S 4}$ has projective unification if and only if $L$ contains $\mathbf{S 4 . 3}$. In particular, we show that these consequence relations enjoy the strong finite model property, and are finitely based. In this way, we extend the known results by Bull and Fine, from logics, to consequence relations. We also show that the lattice of consequence relations over $\mathbf{S 4 . 3}$ (the lattice of quasivarieties of $\mathbf{S 4 . 3}$-algebras) is countable and distributive and it forms a Heyting algebra.
\end{abstract}

\section{Introduction}

Modal logics extending S4.3 form a subset of the lattice of all modal logics in which some theorems on particular logics were generalized to the whole area. Recall that $\mathbf{S 4 . 3}$ is an extension of $\mathbf{S 4}$ with the axiom $\square(\square \alpha \rightarrow \square \beta) \vee \square(\square \beta \rightarrow \square \alpha)$.

First, Bull [4] proved that every such logic has the finite model property, FMP. Next, Fine [11] showed that all modal logics extending $\mathbf{S 4 . 3}$ have the finite frame property, which is equivalent to the FMP, and that they all are finitely axiomatizable. Fine also characterized all such logics by means of finite quasilinear frames (chains of clusters), or lists. Hence he provided a semantic description of all modal logics extending $\mathbf{S 4 . 3}$.

Following this line of investigations, we lift these results from the level of theoremhood into the level of derivability. In other words, we extend these results from

Received November 30, 2012; accepted November 11, 2013

First published online July 19, 2016

2010 Mathematics Subject Classification: Primary 03B45, 08C15, 68T15; Secondary 06E25, 03B35

Keywords: admissible rules, consequence relations, projective unification, $\mathbf{S 4 . 3}$, structural completeness, quasivarieties

(C) 2016 by University of Notre Dame 10.1215/00294527-3636512 


\title{
Modal Consequence Relations Extending S4.3: An Application of Projective Unification
}

\author{
Wojciech Dzik and Piotr Wojtylak
}

\begin{abstract}
We characterize all finitary consequence relations over $\mathbf{S 4 . 3}$, both syntactically, by exhibiting so-called (admissible) passive rules that extend the given logic, and semantically, by providing suitable strongly adequate classes of algebras. This is achieved by applying an earlier result stating that a modal $\operatorname{logic} L$ extending $\mathbf{S 4}$ has projective unification if and only if $L$ contains $\mathbf{S 4 . 3}$. In particular, we show that these consequence relations enjoy the strong finite model property, and are finitely based. In this way, we extend the known results by Bull and Fine, from logics, to consequence relations. We also show that the lattice of consequence relations over $\mathbf{S 4 . 3}$ (the lattice of quasivarieties of $\mathbf{S 4 . 3}$-algebras) is countable and distributive and it forms a Heyting algebra.
\end{abstract}

\section{Introduction}

Modal logics extending S4.3 form a subset of the lattice of all modal logics in which some theorems on particular logics were generalized to the whole area. Recall that $\mathbf{S 4 . 3}$ is an extension of $\mathbf{S 4}$ with the axiom $\square(\square \alpha \rightarrow \square \beta) \vee \square(\square \beta \rightarrow \square \alpha)$.

First, Bull [4] proved that every such logic has the finite model property, FMP. Next, Fine [11] showed that all modal logics extending $\mathbf{S 4 . 3}$ have the finite frame property, which is equivalent to the FMP, and that they all are finitely axiomatizable. Fine also characterized all such logics by means of finite quasilinear frames (chains of clusters), or lists. Hence he provided a semantic description of all modal logics extending $\mathbf{S 4 . 3}$.

Following this line of investigations, we lift these results from the level of theoremhood into the level of derivability. In other words, we extend these results from

Received November 30, 2012; accepted November 11, 2013

First published online July 19, 2016

2010 Mathematics Subject Classification: Primary 03B45, 08C15, 68T15; Secondary 06E25, 03B35

Keywords: admissible rules, consequence relations, projective unification, $\mathbf{S 4 . 3}$, structural completeness, quasivarieties

(C) 2016 by University of Notre Dame 10.1215/00294527-3636512 


\title{
Modal Consequence Relations Extending S4.3: An Application of Projective Unification
}

\author{
Wojciech Dzik and Piotr Wojtylak
}

\begin{abstract}
We characterize all finitary consequence relations over $\mathbf{S 4 . 3}$, both syntactically, by exhibiting so-called (admissible) passive rules that extend the given logic, and semantically, by providing suitable strongly adequate classes of algebras. This is achieved by applying an earlier result stating that a modal $\operatorname{logic} L$ extending $\mathbf{S 4}$ has projective unification if and only if $L$ contains $\mathbf{S 4 . 3}$. In particular, we show that these consequence relations enjoy the strong finite model property, and are finitely based. In this way, we extend the known results by Bull and Fine, from logics, to consequence relations. We also show that the lattice of consequence relations over $\mathbf{S 4 . 3}$ (the lattice of quasivarieties of $\mathbf{S 4 . 3}$-algebras) is countable and distributive and it forms a Heyting algebra.
\end{abstract}

\section{Introduction}

Modal logics extending S4.3 form a subset of the lattice of all modal logics in which some theorems on particular logics were generalized to the whole area. Recall that $\mathbf{S 4 . 3}$ is an extension of $\mathbf{S 4}$ with the axiom $\square(\square \alpha \rightarrow \square \beta) \vee \square(\square \beta \rightarrow \square \alpha)$.

First, Bull [4] proved that every such logic has the finite model property, FMP. Next, Fine [11] showed that all modal logics extending $\mathbf{S 4 . 3}$ have the finite frame property, which is equivalent to the FMP, and that they all are finitely axiomatizable. Fine also characterized all such logics by means of finite quasilinear frames (chains of clusters), or lists. Hence he provided a semantic description of all modal logics extending $\mathbf{S 4 . 3}$.

Following this line of investigations, we lift these results from the level of theoremhood into the level of derivability. In other words, we extend these results from

Received November 30, 2012; accepted November 11, 2013

First published online July 19, 2016

2010 Mathematics Subject Classification: Primary 03B45, 08C15, 68T15; Secondary 06E25, 03B35

Keywords: admissible rules, consequence relations, projective unification, $\mathbf{S 4 . 3}$, structural completeness, quasivarieties

(C) 2016 by University of Notre Dame 10.1215/00294527-3636512 


\title{
Modal Consequence Relations Extending S4.3: An Application of Projective Unification
}

\author{
Wojciech Dzik and Piotr Wojtylak
}

\begin{abstract}
We characterize all finitary consequence relations over $\mathbf{S 4 . 3}$, both syntactically, by exhibiting so-called (admissible) passive rules that extend the given logic, and semantically, by providing suitable strongly adequate classes of algebras. This is achieved by applying an earlier result stating that a modal $\operatorname{logic} L$ extending $\mathbf{S 4}$ has projective unification if and only if $L$ contains $\mathbf{S 4 . 3}$. In particular, we show that these consequence relations enjoy the strong finite model property, and are finitely based. In this way, we extend the known results by Bull and Fine, from logics, to consequence relations. We also show that the lattice of consequence relations over $\mathbf{S 4 . 3}$ (the lattice of quasivarieties of $\mathbf{S 4 . 3}$-algebras) is countable and distributive and it forms a Heyting algebra.
\end{abstract}

\section{Introduction}

Modal logics extending S4.3 form a subset of the lattice of all modal logics in which some theorems on particular logics were generalized to the whole area. Recall that $\mathbf{S 4 . 3}$ is an extension of $\mathbf{S 4}$ with the axiom $\square(\square \alpha \rightarrow \square \beta) \vee \square(\square \beta \rightarrow \square \alpha)$.

First, Bull [4] proved that every such logic has the finite model property, FMP. Next, Fine [11] showed that all modal logics extending $\mathbf{S 4 . 3}$ have the finite frame property, which is equivalent to the FMP, and that they all are finitely axiomatizable. Fine also characterized all such logics by means of finite quasilinear frames (chains of clusters), or lists. Hence he provided a semantic description of all modal logics extending $\mathbf{S 4 . 3}$.

Following this line of investigations, we lift these results from the level of theoremhood into the level of derivability. In other words, we extend these results from

Received November 30, 2012; accepted November 11, 2013

First published online July 19, 2016

2010 Mathematics Subject Classification: Primary 03B45, 08C15, 68T15; Secondary 06E25, 03B35

Keywords: admissible rules, consequence relations, projective unification, $\mathbf{S 4 . 3}$, structural completeness, quasivarieties

(C) 2016 by University of Notre Dame 10.1215/00294527-3636512 


\title{
Modal Consequence Relations Extending S4.3: An Application of Projective Unification
}

\author{
Wojciech Dzik and Piotr Wojtylak
}

\begin{abstract}
We characterize all finitary consequence relations over $\mathbf{S 4 . 3}$, both syntactically, by exhibiting so-called (admissible) passive rules that extend the given logic, and semantically, by providing suitable strongly adequate classes of algebras. This is achieved by applying an earlier result stating that a modal $\operatorname{logic} L$ extending $\mathbf{S 4}$ has projective unification if and only if $L$ contains $\mathbf{S 4 . 3}$. In particular, we show that these consequence relations enjoy the strong finite model property, and are finitely based. In this way, we extend the known results by Bull and Fine, from logics, to consequence relations. We also show that the lattice of consequence relations over $\mathbf{S 4 . 3}$ (the lattice of quasivarieties of $\mathbf{S 4 . 3}$-algebras) is countable and distributive and it forms a Heyting algebra.
\end{abstract}

\section{Introduction}

Modal logics extending S4.3 form a subset of the lattice of all modal logics in which some theorems on particular logics were generalized to the whole area. Recall that $\mathbf{S 4 . 3}$ is an extension of $\mathbf{S 4}$ with the axiom $\square(\square \alpha \rightarrow \square \beta) \vee \square(\square \beta \rightarrow \square \alpha)$.

First, Bull [4] proved that every such logic has the finite model property, FMP. Next, Fine [11] showed that all modal logics extending $\mathbf{S 4 . 3}$ have the finite frame property, which is equivalent to the FMP, and that they all are finitely axiomatizable. Fine also characterized all such logics by means of finite quasilinear frames (chains of clusters), or lists. Hence he provided a semantic description of all modal logics extending $\mathbf{S 4 . 3}$.

Following this line of investigations, we lift these results from the level of theoremhood into the level of derivability. In other words, we extend these results from

Received November 30, 2012; accepted November 11, 2013

First published online July 19, 2016

2010 Mathematics Subject Classification: Primary 03B45, 08C15, 68T15; Secondary 06E25, 03B35

Keywords: admissible rules, consequence relations, projective unification, $\mathbf{S 4 . 3}$, structural completeness, quasivarieties

(C) 2016 by University of Notre Dame 10.1215/00294527-3636512 


\title{
Modal Consequence Relations Extending S4.3: An Application of Projective Unification
}

\author{
Wojciech Dzik and Piotr Wojtylak
}

\begin{abstract}
We characterize all finitary consequence relations over $\mathbf{S 4 . 3}$, both syntactically, by exhibiting so-called (admissible) passive rules that extend the given logic, and semantically, by providing suitable strongly adequate classes of algebras. This is achieved by applying an earlier result stating that a modal $\operatorname{logic} L$ extending $\mathbf{S 4}$ has projective unification if and only if $L$ contains $\mathbf{S 4 . 3}$. In particular, we show that these consequence relations enjoy the strong finite model property, and are finitely based. In this way, we extend the known results by Bull and Fine, from logics, to consequence relations. We also show that the lattice of consequence relations over $\mathbf{S 4 . 3}$ (the lattice of quasivarieties of $\mathbf{S 4 . 3}$-algebras) is countable and distributive and it forms a Heyting algebra.
\end{abstract}

\section{Introduction}

Modal logics extending S4.3 form a subset of the lattice of all modal logics in which some theorems on particular logics were generalized to the whole area. Recall that $\mathbf{S 4 . 3}$ is an extension of $\mathbf{S 4}$ with the axiom $\square(\square \alpha \rightarrow \square \beta) \vee \square(\square \beta \rightarrow \square \alpha)$.

First, Bull [4] proved that every such logic has the finite model property, FMP. Next, Fine [11] showed that all modal logics extending $\mathbf{S 4 . 3}$ have the finite frame property, which is equivalent to the FMP, and that they all are finitely axiomatizable. Fine also characterized all such logics by means of finite quasilinear frames (chains of clusters), or lists. Hence he provided a semantic description of all modal logics extending $\mathbf{S 4 . 3}$.

Following this line of investigations, we lift these results from the level of theoremhood into the level of derivability. In other words, we extend these results from

Received November 30, 2012; accepted November 11, 2013

First published online July 19, 2016

2010 Mathematics Subject Classification: Primary 03B45, 08C15, 68T15; Secondary 06E25, 03B35

Keywords: admissible rules, consequence relations, projective unification, $\mathbf{S 4 . 3}$, structural completeness, quasivarieties

(C) 2016 by University of Notre Dame 10.1215/00294527-3636512 


\title{
Modal Consequence Relations Extending S4.3: An Application of Projective Unification
}

\author{
Wojciech Dzik and Piotr Wojtylak
}

\begin{abstract}
We characterize all finitary consequence relations over $\mathbf{S 4 . 3}$, both syntactically, by exhibiting so-called (admissible) passive rules that extend the given logic, and semantically, by providing suitable strongly adequate classes of algebras. This is achieved by applying an earlier result stating that a modal $\operatorname{logic} L$ extending $\mathbf{S 4}$ has projective unification if and only if $L$ contains $\mathbf{S 4 . 3}$. In particular, we show that these consequence relations enjoy the strong finite model property, and are finitely based. In this way, we extend the known results by Bull and Fine, from logics, to consequence relations. We also show that the lattice of consequence relations over $\mathbf{S 4 . 3}$ (the lattice of quasivarieties of $\mathbf{S 4 . 3}$-algebras) is countable and distributive and it forms a Heyting algebra.
\end{abstract}

\section{Introduction}

Modal logics extending S4.3 form a subset of the lattice of all modal logics in which some theorems on particular logics were generalized to the whole area. Recall that $\mathbf{S 4 . 3}$ is an extension of $\mathbf{S 4}$ with the axiom $\square(\square \alpha \rightarrow \square \beta) \vee \square(\square \beta \rightarrow \square \alpha)$.

First, Bull [4] proved that every such logic has the finite model property, FMP. Next, Fine [11] showed that all modal logics extending $\mathbf{S 4 . 3}$ have the finite frame property, which is equivalent to the FMP, and that they all are finitely axiomatizable. Fine also characterized all such logics by means of finite quasilinear frames (chains of clusters), or lists. Hence he provided a semantic description of all modal logics extending $\mathbf{S 4 . 3}$.

Following this line of investigations, we lift these results from the level of theoremhood into the level of derivability. In other words, we extend these results from

Received November 30, 2012; accepted November 11, 2013

First published online July 19, 2016

2010 Mathematics Subject Classification: Primary 03B45, 08C15, 68T15; Secondary 06E25, 03B35

Keywords: admissible rules, consequence relations, projective unification, $\mathbf{S 4 . 3}$, structural completeness, quasivarieties

(C) 2016 by University of Notre Dame 10.1215/00294527-3636512 


\title{
Modal Consequence Relations Extending S4.3: An Application of Projective Unification
}

\author{
Wojciech Dzik and Piotr Wojtylak
}

\begin{abstract}
We characterize all finitary consequence relations over $\mathbf{S 4 . 3}$, both syntactically, by exhibiting so-called (admissible) passive rules that extend the given logic, and semantically, by providing suitable strongly adequate classes of algebras. This is achieved by applying an earlier result stating that a modal $\operatorname{logic} L$ extending $\mathbf{S 4}$ has projective unification if and only if $L$ contains $\mathbf{S 4 . 3}$. In particular, we show that these consequence relations enjoy the strong finite model property, and are finitely based. In this way, we extend the known results by Bull and Fine, from logics, to consequence relations. We also show that the lattice of consequence relations over $\mathbf{S 4 . 3}$ (the lattice of quasivarieties of $\mathbf{S 4 . 3}$-algebras) is countable and distributive and it forms a Heyting algebra.
\end{abstract}

\section{Introduction}

Modal logics extending S4.3 form a subset of the lattice of all modal logics in which some theorems on particular logics were generalized to the whole area. Recall that $\mathbf{S 4 . 3}$ is an extension of $\mathbf{S 4}$ with the axiom $\square(\square \alpha \rightarrow \square \beta) \vee \square(\square \beta \rightarrow \square \alpha)$.

First, Bull [4] proved that every such logic has the finite model property, FMP. Next, Fine [11] showed that all modal logics extending $\mathbf{S 4 . 3}$ have the finite frame property, which is equivalent to the FMP, and that they all are finitely axiomatizable. Fine also characterized all such logics by means of finite quasilinear frames (chains of clusters), or lists. Hence he provided a semantic description of all modal logics extending $\mathbf{S 4 . 3}$.

Following this line of investigations, we lift these results from the level of theoremhood into the level of derivability. In other words, we extend these results from

Received November 30, 2012; accepted November 11, 2013

First published online July 19, 2016

2010 Mathematics Subject Classification: Primary 03B45, 08C15, 68T15; Secondary 06E25, 03B35

Keywords: admissible rules, consequence relations, projective unification, $\mathbf{S 4 . 3}$, structural completeness, quasivarieties

(C) 2016 by University of Notre Dame 10.1215/00294527-3636512 


\title{
Modal Consequence Relations Extending S4.3: An Application of Projective Unification
}

\author{
Wojciech Dzik and Piotr Wojtylak
}

\begin{abstract}
We characterize all finitary consequence relations over $\mathbf{S 4 . 3}$, both syntactically, by exhibiting so-called (admissible) passive rules that extend the given logic, and semantically, by providing suitable strongly adequate classes of algebras. This is achieved by applying an earlier result stating that a modal $\operatorname{logic} L$ extending $\mathbf{S 4}$ has projective unification if and only if $L$ contains $\mathbf{S 4 . 3}$. In particular, we show that these consequence relations enjoy the strong finite model property, and are finitely based. In this way, we extend the known results by Bull and Fine, from logics, to consequence relations. We also show that the lattice of consequence relations over $\mathbf{S 4 . 3}$ (the lattice of quasivarieties of $\mathbf{S 4 . 3}$-algebras) is countable and distributive and it forms a Heyting algebra.
\end{abstract}

\section{Introduction}

Modal logics extending S4.3 form a subset of the lattice of all modal logics in which some theorems on particular logics were generalized to the whole area. Recall that $\mathbf{S 4 . 3}$ is an extension of $\mathbf{S 4}$ with the axiom $\square(\square \alpha \rightarrow \square \beta) \vee \square(\square \beta \rightarrow \square \alpha)$.

First, Bull [4] proved that every such logic has the finite model property, FMP. Next, Fine [11] showed that all modal logics extending $\mathbf{S 4 . 3}$ have the finite frame property, which is equivalent to the FMP, and that they all are finitely axiomatizable. Fine also characterized all such logics by means of finite quasilinear frames (chains of clusters), or lists. Hence he provided a semantic description of all modal logics extending $\mathbf{S 4 . 3}$.

Following this line of investigations, we lift these results from the level of theoremhood into the level of derivability. In other words, we extend these results from

Received November 30, 2012; accepted November 11, 2013

First published online July 19, 2016

2010 Mathematics Subject Classification: Primary 03B45, 08C15, 68T15; Secondary 06E25, 03B35

Keywords: admissible rules, consequence relations, projective unification, $\mathbf{S 4 . 3}$, structural completeness, quasivarieties

(C) 2016 by University of Notre Dame 10.1215/00294527-3636512 